REZENSIONEN

\section{Wissenschaftskommunikation am Beispiel des Klimawandels}

\author{
H. P. Peters, H. Heinrichs: Öffentliche \\ Kommunikation über Klimawandel und \\ Sturmflutrisiken. Bedeutungskonstruk- \\ tion durch Experten, Journalisten und \\ Bürger. Jülich: Forschungszentrums \\ Jülich, 2005 (Reihe Umwelt, Band 58), \\ 231 S., ISBN 3-89336-415-3, EURO 49,00

\section{Rezension von Jürgen Hampel, Universität Stuttgart}

Der Klimawandel gehört zu den nicht direkt wahrnehmbaren Risiken. Es bedarf der Vermittlung von Informationen aus der Wissenschaft, damit dieses Risiko von der Öffentlichkeit überhaupt wahrgenommen werden kann. Um diesen Vermittlungsprozess, der überwiegend in den Medien geführt wird, geht es in dem von Hans Peter Peters und Harald Heinrichs verfassten Buch, in dem über die Kommunikation über den Klimawandel aus der Sicht von Experten, Journalisten und Bürgern berichtet wird.

\section{Hintergrund}

Das vom BMBF geförderte interdisziplinäre Verbundvorhaben ,Klimawandel und präventives Risiko- und Küstenschutzmanagement an der deutschen Nordseeküste" (KRIM) hat im Rahmen der Klimawirkungsforschung die Gefährdungen der deutschen Küsten durch den Klimawandel untersucht. Da der Klimawandel zu den für die Öffentlichkeit nicht direkt wahrnehmbaren Risiken gehört, die von Experten in die Öffentlichkeit getragen werden müssen, wurde im Rahmen dieses Projektverbunds auch ein sozialwissenschaftliches Teilprojekt finanziert, das sich mit den Risikowahrnehmungen der Öffentlichkeit und den Prozessen beschäftigte, die diese Wahrnehmungen beeinflussen,.

Bei dem vorliegenden Buch handelt es sich um den von den beiden Sozialwissenschaftlern Peters und Heinrichs ausgearbeiteten Abschlussbericht dieses Teilprojekts. Die vorliegende Rezension stellt das Untersuchungs- design und die gewählten Analysemethoden vor, fasst die Ergebnisse der Studie zusammen und bewertet deren wissenschaftlichen Ertrag.

\section{Das Untersuchungsdesign}

Peters und Heinrichs untersuchen die Risiken in Bezug auf den globalen Klimawandel (Klimarisiken) und die Sturmflut-Risiken für die Nordseeküsten (Küstenrisiken) auf drei Ebenen: (1) als mediale Repräsentation, (2) als kognitive Reaktionen, die bei der Rezeption beispielhaft ausgewählter Artikel ausgelöst wurden und (3) als Risikobewusstsein der an der Küste wohnenden Bevölkerung. In ihrer Untersuchung haben sie für jede dieser drei Ebenen eigene empirische Erhebungen durchgeführt. Das außergewöhnlich komplexe methodische Design umfasst

- die Analyse thematisch relevanter regionaler und überregionaler Medienberichterstattung in Printmedien, Hörfunk und Fernsehen anhand von insgesamt 1.176 Medienbeiträgen („Medienanalyse“),

- eine Befragung von 183 Küstenbewohnern,

- eine Analyse der bei der Rezeption von einschlägigen Medienberichten auftretenden Risikoreferenzen. Dazu sollten von den 183 Interviewpartnern jeweils vier kurze Zeitungsartikel über Klima- und Küstenrisiken durchgelesen werden. Die kognitiven Reaktionen, die die Testleser bei der Lektüre dieser Artikel äußerten, wurden erfasst, was insgesamt 732 Gedankenprotokolle ergab („,Rezeptionsstudie“),

- eine Untersuchung der Bedingungen der Wissenschaftskommunikation. Dazu wurden neben 85 Journalisten, die Medienberichte über Klima- und Küstenrisiken verfasst haben, 169 Experten befragt, zu denen nicht nur Fachwissenschaftler, sondern auch Praxis-Experten und Vertreter von NGOs zählten („Interaktionsstudie“).

\section{Ergebnisse der Medienanalyse}

Die Autoren beginnen mit der Analyse der Medienberichterstattung, bei der sie eindeutige Unterschiede zwischen der lokalen und regionalen und der überregionalen Presse beobachten. Während die überregionale Presse vor allem über Klimarisiken berichtet und Küstenrisiken 
nur in diesem Kontext behandelt, werden von der lokalen und regionalen Presse beide Risikoarten thematisiert. In der Berichterstattung über Klima- und Küstenrisiken ist eine eindeutige Tendenz feststellbar: Nur ein geringer Anteil der Beiträge über diese Themen ist ,beruhigend“, der Löwenanteil hat entweder ,keine eindeutige Tendenz" oder ist sogar „alarmierend“.

Wenn man die Primärquellen für Risikoinformationen betrachtet, erweist sich die Wissenschaft als dominant. Die meisten Aussagen über den jeweiligen Zusammenhang von Ursache und Wirkung, für die eine Primärquelle angegeben ist, stammen aus der Wissenschaft (S. 57). Rund die Hälfte der Risikoinformationen sind auf Wissenschaft und Technik zurückzuführen, in überregionalen Medien noch stärker (52\%) als in regionalen Medien (38 \%). Mit weitem Abstand folgt Politik / Administration als zweitwichtigste Primärquelle von Risikoinformationen.

Als mögliche Maßnahmen zur Bewältigung des Klimawandels werden von den Medien so unterschiedliche Aktionen wie technische und soziale Innovationen, internationale Übereinkommen zum Klimaschutz, die Verbesserung der Deiche sowie nationale politische Maßnahmen und Reaktionen genannt.

\section{Ergebnisse der Rezeptionsstudie}

Für die Küstenanwohner selbst sind Klima- und Küstenrisiken evident. Bei der Lektüre der Medienbeiträge werden diese Risiken kaum bestritten oder heruntergespielt, sondern als ernste Bedrohung betrachtet. Dabei folgen die Gedanken der Testpersonen weitgehend den von den Artikeln vorgegebenen Themen. Dass die These des anthropogen erzeugten Klimawandels zum „common sense“ gehört, führt dazu, dass Gegenargumente gegen diese These explizit zurückgewiesen werden, so ein Ergebnis der Studie. Dies werde an den negativen kognitiven Reaktionen auf einen Artikel deutlich, der die gängige These zurückweist, Treibhausgase verursachten den Klimawandel. Das gleiche Phänomen zeigt sich bei der Bewertung der Wissenschaftler, auf die in den Artikeln Bezug genommen wird. Wissenschaftler erhalten zwar die positivsten Bewertungen - allerdings nur, solange die von Wissenschaftlern vertretene Position mit der Position der Urteilenden übereinstimmt.
Wissenschaftler, die dem ,common sense“ widersprechen, werden dagegen negativ beurteilt.

Nicht nur die Existenz des Klimawandels, sondern auch seine anthropogene Verursachung ist den Ergebnissen von Peters und Heinrichs zufolge allgemein anerkannter gesellschaftlicher Konsens. In ihren kognitiven Reaktionen auf die Medienberichte machen die Befragten auch nicht Industrie oder Politik für den Klimawandels verantwortlich, sondern die Bevölkerung selbst. Die Einschätzung, dass der Klimawandel von der Bevölkerung selbst zu verantworten ist, führt auf den ersten Blick zu einer erheblichen Handlungsbereitschaft: Für rund 70 Prozent der Befragten ist der Klimawandel nach eigener Einschätzung ein Grund, freiwillig Abstriche vom Lebensstandard $\mathrm{zu}$ machen. Ob diese bekundete Bereitschaft sich tatsächlich in Handlungen äußern wird, bleibt allerdings ungewiss. Vieles deutet darauf hin, dass hier Skepsis angebracht ist. So wird die Ökosteuer, die einzig konkrete Maßnahme zur Bekämpfung des Klimawandels, die in der Studie genannt wird, nur von der Hälfte der Befragten unterstützt.

\section{Ergebnisse der Interaktionsstudie}

Dass es die wissenschaftliche These vom anthropogen verursachten Klimawandel in das Bewusstsein der Öffentlichkeit geschafft hat, spricht für eine ausgesprochen erfolgreiche Kommunikation zwischen Wissenschaft und Öffentlichkeit. Diese Kommunikation wird in der Interaktionsstudie genauer untersucht.

Erfolgreiche Kommunikation zwischen "Experten" (Fachwissenschaftlern, Experten aus der Praxis und Vertretern von NGOs) und Journalisten ist alles andere als trivial. Die Erwartungen an die Kommunikation könnten größer kaum sein (S. 99). So erwarten Journalisten von Experten, dass diese sich bereits äußern, bevor es einen eindeutigen wissenschaftlichen Konsens gibt und dabei auch bereit sind, Entscheidungen zu kritisieren und Handlungsvorschläge zu machen. Auf der anderen Seite schreiben Experten dem Journalismus stärker eine paternalistisch-volkserziehende Funktion zu, die von Journalisten wiederum zurückgewiesen wird. Missverständnisse scheinen daher vorprogrammiert.

Umso überraschender ist das Ergebnis, dass es zwischen Experten und Journalisten 
kaum Kommunikationsbarrieren $\mathrm{zu}$ geben scheint (S.129). Die überwiegende Mehrheit (79 \%) der Experten bewertet ihre Medienerfahrungen sogar als ,überwiegend gut“. Noch besser urteilen die Journalisten über die Experten. Hier berichten neun von zehn Journalisten, dass ihre Erfahrungen mit Experten ,überwiegend gut" seien. Nur wenige Kritikpunkte werden genannt. So bemängeln einige Experten die zum Teil schlechte inhaltliche Vorbereitung von Journalisten. Prinzipieller ist aber der Konflikt um die Kontrolle der Kommunikation. Während von Experten eine Überprüfung der Berichte eingefordert wird, lehnen Journalisten dies als Eingriff in ihre Sphäre ab.

Da die Autoren nur Journalisten befragt haben, die von den Medien nachgefragt wurden, weisen die Autoren darauf hin, dass sich methodisch nicht feststellen lässt, ob Journalisten bei der Auswahl der von ihnen nachgefragten Wissenschaftler selektiv vorgehen. Für Selektionsprozesse spricht nach Auffassung der Autoren, dass es Hinweise gibt, wonach Experten, die politisch wirken wollen, eher nachgefragt würden als andere Experten.

Die meisten Experten, diejenigen aus der Praxis eher noch als die Fachwissenschaftler, haben bereits umfangreiche Erfahrungen im Umgang mit Medien. Eine ganze Reihe von ihnen wurde in den letzten zwölf Monaten vor dem Interview 50-mal und öfter in den Medien erwähnt. Typisch seien mehrere Erwähnungen in den Printmedien sowie einige Hörfunk- und Fernsehauftritte. Die enge Verbindung zwischen Journalisten und zumindest einem Teil der Experten wirft die Frage auf, wie die Kontakte zwischen beiden Gruppen zustande kommen. Wie Peters und Heinrichs zeigen, geht die Initiative hier in aller Regel von den Journalisten aus, nur sehr selten ergreifen Experten die Initiative und gehen von sich aus auf Journalisten zu. Ein Teil der Kontakte kam aber auch durch die Öffentlichkeitsabteilungen wissenschaftlicher Einrichtungen zustande. Von Journalisten werden dabei vor allem solche Experten kontaktiert, die als Autoren von Gutachten oder hochrangigen Veröffentlichungen sichtbar geworden sind, wobei deren Medienkompetenz weniger bedeutsam für die Journalisten zu sein scheint als der wissenschaftliche Status und die wahrgenommenen Kompetenz des Experten. Weniger überraschend ist das Ergebnis, dass Wissenschaftler, die politisch wirken wollen, eher bereit sind, mit Journalisten zusammenzuarbeiten, während Journalisten umgekehrt Experten bevorzugen, die nicht nur über wissenschaftliche Sachverhalte, sondern auch über politische Perspektiven Auskunft geben.

\section{Empfehlungen für die öffentliche Kommunikation}

Am Ende runden Empfehlungen für die öffentliche Risiko- und Umweltkommunikation das Buch ab. Obwohl es allgemeiner Konsens ist, dass der Klimawandel anthropogen verursacht wird, scheint es sich hier eher um eine abstrakte Einschätzung zu handeln als um etwas, von dem direkte Auswirkungen auf das eigene Leben erwartet werden. Daher ist es nicht überraschend, dass von den Befragten eher konservative Maßnahmen bevorzugt werden, die direkt an die gängigen Küstenschutzstrategien anschließen. Wenn diese konservativen Maßnahmen des Küstenschutzes nicht ausreichen, halten die Autoren einen intensiven Dialog mit der Bevölkerung für erforderlich. Eine völlige Neuausrichtung des Küstenschutzes wäre nach Einschätzung der Autoren aber erst nach krisenhaften Ereignissen durchsetzbar.

\section{Fazit}

Die Studie von Peters und Heinrichs bietet eine umfassende Bearbeitung der sozialen und medialen Konstruktion von Küstenrisiken. Hervorzuheben ist vor allem das komplexe methodische Design, das es erlaubt, die Fragestellung sehr facettenreich aus unterschiedlichen Perspektiven mit selbst erhobenen Primärdaten zu untersuchen. Hochinteressant ist auch der Teil der Studie, der sich mit der Interaktion zwischen Experten und Journalisten beschäftigt. Den Materialreichtum des Buches erkennt man auch daran, dass im Text 88 Tabellen vorgestellt und erläutert werden. Die Zusammenfassung der wichtigsten Ergebnisse am Ende jedes Kapitels trägt zur besseren Lesbarkeit auch für nicht-wissenschaftliche Nutzer bei. In Anbetracht der Qualitäten der Studie und des Buches ist es nur von untergeordneter Bedeutung, dass man sich mitunter eine etwas straffere Form der Darstellung wünschen würde. 